\title{
RAS-associated autoimmune leukoproliferative disease
}

INSERM

\section{Source}

INSERM. (1999). Orphanet: an online rare disease and orphan drug data base. RAS-

associated autoimmune leukoproliferative disease. ORPHA:268114

RAS-associated autoimmune leukoproliferative disease (RALD) is a rare genetic disorder characterized by monocytosis, autoimmune cytopenias, lymphoproliferation, hepatosplenomegaly, and hypergammaglobulinemia. 